\title{
Multiperiodic pulsations in the Be stars NW Serpentis and V1446 Aquilae ${ }^{\star}$
}

\author{
J. Gutiérrez-Soto ${ }^{1,5}$, J. Fabregat ${ }^{1}$, J. Suso ${ }^{2}$, J. C. Suárez ${ }^{3}$, A. Moya ${ }^{3,4}$, R. Garrido ${ }^{3}$, \\ A.-M. Hubert ${ }^{5}$, M. Floquet ${ }^{5}$, C. Neiner ${ }^{5}$, and Y. Frémat ${ }^{6}$
}

1 Observatorio Astronómico. Universidad de Valencia. Edificio Institutos de investigación. Polígono la Coma, 46980 Paterna, Valencia, Spain e-mail: juan.gutierrez-soto@uv.es

2 ICMUV, Edificio Institutos de investigación, Polígono la Coma, 46980 Paterna, Valencia, Spain

Instituto de Astrofísica de Andalucía (CSIC) Camino Bajo de Huétor, 24, 18008 Granada, Spain

4 LESIA, UMR 8109 du CNRS, Observatoire de Paris-Meudon, 92195 Meudon, France

5 GEPI, UMR 8111 du CNRS, Observatoire de Paris-Meudon, 92195 Meudon, France

${ }^{6}$ Royal Observatory of Belgium, 3 avenue Circulaire, 1180 Brussels, Belgium

Received 5 March 2007 / Accepted 6 June 2007

\section{ABSTRACT}

\begin{abstract}
Aims. We present accurate photometric time series of two Be stars: NW Ser and V1446 Aql. Both stars were observed at the Observatorio de Sierra Nevada (Granada) in July 2003 with an automatic four-channel Strömgren photometer. We also present a preliminary theoretical study showing that the periodic variations exhibited by these stars can be due to pulsation.

Methods. An exhaustive Fourier analysis together with a least-square fitting has been carried out on the time series for all four Strömgren bands. Several independent frequencies and non-periodic trends explain most of the variance. A theoretical non-adiabatic code applied to stellar models for these stars shows that g-modes are unstable.

Results. Both stars show rapid variations in amplitude, probably due to a beating phenomenon. Four significant frequencies have been detected for each star. Comparison of the observed amplitude ratios for each pulsational frequency with those calculated from theoretical pulsation codes allows us to estimate the pulsation modes associated with the different detected frequencies. NW Ser seems also to show unstable p-modes and thus could be one of the newly discovered $\beta$ Cephei and SPB hybrid stars. Further spectroscopic observations are planned to study the stability of the detected frequencies.
\end{abstract}

Key words. stars: oscillations - stars: emission-line, Be - stars: individual: NW Serpentis - stars: individual: V1446 Aquilae

\section{Introduction}

Short-term variability, with periods ranging from a few hours to two days, is present in most of the early-type Be stars and in a significant fraction of late Be stars. It has been detected both spectroscopically, in the form of line profile variability $(l p v)$ and photometrically, by means of non-regular light curves.

Baade (1982) attributed the short periodic $l p v$ to non-radial pulsations ( $n r p)$. Further detailed studies of a few well-observed objects led to a complete and consistent multimode modelling of the observed variations (Rivinius et al. 2001; Maintz et al. 2003; Neiner et al. 2005). In addition, high-precision photometric data obtained with the MOST satellite showed the presence of multiple periods in the Be stars $\zeta$-Oph (Walker et al. 2005b) and HD 163868 (Walker et al. 2005a). They modelled the detected frequencies as $n r p$ in terms of $\mathrm{p}$-modes in the case of $\zeta$-Oph, and g- and r-modes in the case of HD 163868.

Similarly, COROT will provide important clues to understand the link between the beating of $n r p$ modes and the origin of the Be phenomenon. COROT is based on ultra high

* Tables A.1 and A.2 are only available in electronic form at the CDS via anonymous ftp to cdsarc.u-strasbg. $f r(130.79 .128 .5)$ or via http://cdsweb.u-strasbg.fr/cgi-bin/qcat? J/A+A/472/565 precision, wide field photometry for very long continuous observing runs in the same field of view (Baglin et al. 2002). In preparation for this mission, a total of 84 Be stars have been studied photometrically in order to select the most suitable candidates to be observed by COROT (Gutiérrez-Soto et al., in preparation). Here we present the analysis of the photometric time series for two Be stars, namely NW Ser and V1446 Aql.

NW Ser (HR 6873, HD 168797, $V=6.14$ ) is a bright and extensively observed B2.5IIIe star, which has been photometrically monitored for over two decades. From the analysis of the Hipparcos photometric data, several authors have obtained similar results: Hubert \& Floquet (1998) found short-term variability with a period of 0.488 days; Percy et al. (1999) reanalysed the Hipparcos data together with ground-based photometry finding a similar period of 0.46 days, as well as a longer period of 5.5 days; and finally, two periods ( 0.475 and 0.406 days) have been detected by Aerts (2000) using only Hipparcos data, although the author claimed that this finding should be confirmed by means of ground-based observations.

V1446 Aql (HD 179405, B2IVe) has been observed to be an emission-line star in the Mount Wilson objective prism allsky survey (Merrill \& Burwell 1943). It appears as an irregular variable star from the Hipparcos data, which led to its inclusion 
Table 1. Comparison and check stars used in the differential photometry.

\begin{tabular}{cccc}
\hline \hline & Star & $V$ & Spectral type \\
\hline Variable & NW Ser & 6.14 & B2.5IIIe \\
Comparison & HD 170 200 & 5.71 & B8III-IV \\
Check & SAO 123607 & 8.6 & B8 \\
\hline Variable & V1446 Aql & 9.12 & B2IVe \\
Comparison & HD 179 846 & 8.29 & B8 \\
Check & HD 178598 & 9.45 & B8 \\
\hline
\end{tabular}

Table 2. Photometric precision in the instrumental system.

\begin{tabular}{ccccc}
\hline \hline Star & $u$ filter & $v$ filter & $b$ filter & $y$ filter \\
\hline NW Ser & 0.010 & 0.005 & 0.005 & 0.005 \\
V1446 Aql & 0.011 & 0.007 & 0.007 & 0.008 \\
\hline
\end{tabular}

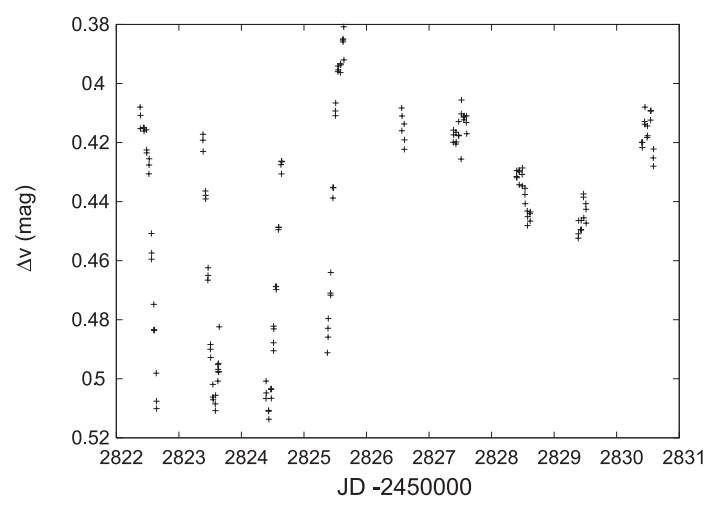

Fig. 1. Light curve of NW Ser in the $v$ filter.

in the GCVS (Kazarovets et al. 1999), although no short-term periodic variability had been detected so far.

\section{Observations and frequency analysis}

Observations were made with the $0.9 \mathrm{~m}$ telescope of the Observatorio de Sierra Nevada (OSN, Granada, Spain) between July 1 and 9 in 2003. The instrument used is an automatic four-channel photometer, which allows simultaneous observations through the four uvby filters of the Strömgren photometric system. The data discussed here and presented in Tables A.1 and A.2 are the differential magnitude in the instrumental system between the variable and the comparison star of Table 1 . A check star has also been observed to verify possible intrinsic variations of the comparison star. Data have been corrected for sky background and atmospheric extinction. Table 2 shows the photometric precision as given by the standard deviation of the difference between the comparison and check stars for the whole campaign for each filter. The light curves of the stars are depicted in Figs. 1 and 3, only the $v$ filter data are shown for clarity.

The period analysis has been performed by means of standard Fourier analysis and least-square fitting. We have used Period04 (Lenz \& Breger 2005) which is especially designed for the analysis of time series containing gaps. This program finds the frequencies one by one by computing the Fourier Transform and then adjusts the parameters of a sinusoidal function using a least-square fitting. This frequency is then removed and a new step is started finding a new frequency, the subsequent leastsquare fitting is performed allowing the two frequencies to move in order to obtain the minimum variance. The method is iterative
Table 3. Frequencies, amplitudes and phases obtained with the multiparameter fitting code for NW Ser for the four Strömgren bands. The SNR, the $\sigma$ of the final residual $\left(\sigma_{\text {res }}\right)$ and the total fraction of the variance removed from the signal $(R)$ are also given.

\begin{tabular}{|c|c|c|c|c|c|c|}
\hline No. & $\begin{array}{l}\text { Freq. } \\
\mathrm{c} \mathrm{d}^{-1}\end{array}$ & $\begin{array}{l}\text { Amp. } \\
\text { mmag }\end{array}$ & $\begin{array}{l}\text { Phase } \\
2 \pi \text { rad }\end{array}$ & $\overline{\mathrm{SNR}}$ & $\begin{array}{c}\sigma_{\text {res }} \\
\text { mmag }\end{array}$ & $\begin{array}{l}R \\
\%\end{array}$ \\
\hline & Filter $y$ & & $N=162$ & \multicolumn{3}{|c|}{$\sigma_{\text {init }}=34.8 \mathrm{mmag}$} \\
\hline$F 1$ & 1.190 & 38.1 & 0.84 & 25 & 20.8 & \\
\hline$F 2$ & 1.119 & 21.8 & 0.88 & 15 & 12.1 & \\
\hline$F 3$ & 3.294 & 13.6 & 0.38 & 7 & 8.7 & \\
\hline$F 4$ & 1.415 & 7.8 & 0.29 & 5 & 7.1 & 96 \\
\hline & Filter $b$ & & $N=162$ & \multicolumn{3}{|c|}{$\sigma_{\text {init }}=34.9 \mathrm{mmag}$} \\
\hline$F 1$ & 1.196 & 36.6 & 0.85 & 25 & 22.0 & \\
\hline$F 2$ & 1.127 & 26.9 & 0.86 & 19 & 11.7 & \\
\hline$F 3$ & 3.301 & 11.6 & 0.36 & 7 & 8.7 & \\
\hline$F 4$ & 1.414 & 9.5 & 0.28 & 6 & 6.2 & 97 \\
\hline & Filter $v$ & & $N=162$ & \multicolumn{3}{|c|}{$\sigma_{\text {init }}=35.1 \mathrm{mmag}$} \\
\hline$F 1$ & 1.197 & 36.4 & 0.86 & 28 & 22.6 & \\
\hline$F 2$ & 1.126 & 27.7 & 0.88 & 22 & 12.1 & \\
\hline$F 3$ & 3.304 & 11.3 & 0.30 & 7 & 8.5 & \\
\hline$F 4$ & 1.412 & 10.5 & 0.36 & 7 & 6.2 & 97 \\
\hline & Filter $u$ & & $N=162$ & \multicolumn{3}{|c|}{$\sigma_{\text {init }}=44.4 \mathrm{mmag}$} \\
\hline$F 1$ & 1.159 & 55.6 & 0.95 & 38 & 26.2 & \\
\hline$F 2$ & 1.103 & 33.3 & 0.83 & 23 & 14.2 & \\
\hline$F 3$ & 2.274 & 17.5 & 0.49 & 11 & 10.5 & \\
\hline$F 4$ & 2.387 & 12.9 & 0.34 & 8 & 7.4 & 97 \\
\hline
\end{tabular}

and stops when removing of a new frequency is not statistically significant.

We also used a non-linear multi-parameter fitting code which scans a wide range in frequency based on Vaníček (1971) and is explained in detail in Zerbi et al. (1997). This code is also well-suited for our case, for which daily aliases are present in the periodogram, due to the fact that our observations have been obtained at only one site.

The way in which we determine whether the frequencies are statistically significant or not is described in Breger et al. (1993) and basically consists of the calculation of the signal to noise ratio (SNR), the noise being the average amplitude, within a $5 \mathrm{c} \mathrm{d}^{-1}$ frequency interval, of the residual periodogram after the prewhitening as previously explained around a frequency and the signal being the corresponding amplitude of that frequency. Breger et al. (1993) showed that this value must be greater than 4.

Frequencies, amplitudes and phases obtained for NW Ser and V1446 Aql are presented in Tables 3 and 4 respectively for the four Strömgren bands. We also show the total fraction of the variance removed from the signal $(R)$, the SNR and the $\sigma$ of the final residual $\sigma_{\text {res }}$. In Figs. 2 and 4 we display the successive periodograms and the spectral window for NW Ser and V1446 Aql respectively, results are shown only for the $v$ filter data for clarity.

Following Montgomery \& O'Donoghue (1999), the expected error in frequency for uncorrelated observations can be derived from the equation

$\sigma_{F}=\frac{\sqrt{6}}{\pi} \cdot \frac{\sigma_{n}}{A \cdot \sqrt{N} \cdot T}$

where $A / \sigma_{n}$ indicates the SNR, $N$ is the number of observations and $T$ the time elapsed between the first and the last data point. As noted by Schwarzenberg-Czerny (1991), correlations in the residuals of the fitting have to be taken into account multiplying the error frequency by $\sqrt{D}$, where $D$ is the correlation length. $D$ can be estimated by performing an autocorrelation analysis of the final residuals. In our case the correlation 

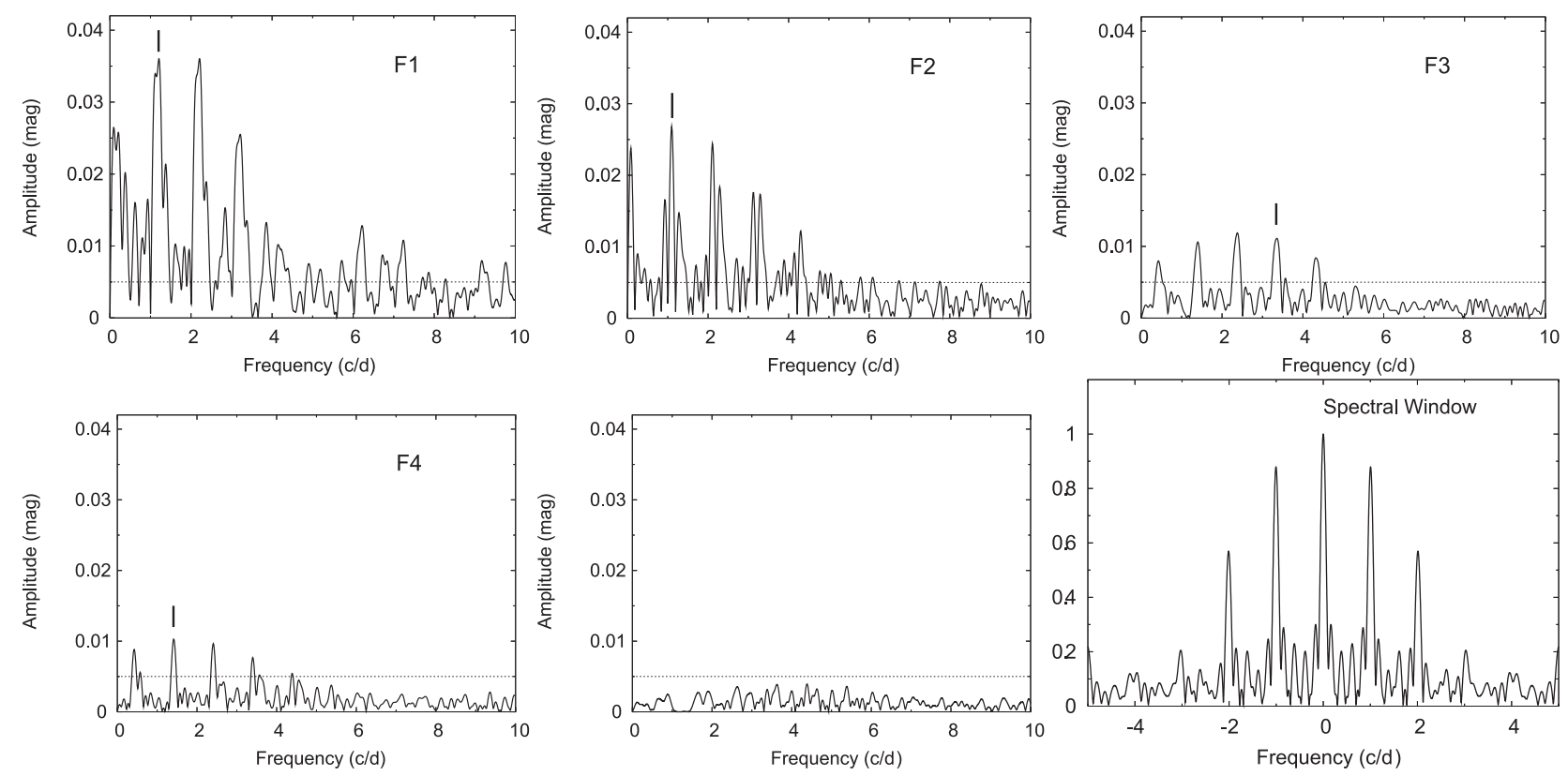

Fig. 2. Successive periodograms for NW Ser for the $v$ filter. The ticks indicate the positions of the detected frequencies and the dashed horizontal line indicates the $4 \sigma$ level after the final prewhitening.

length is 4 . Therefore, we derive a formal error in frequency of $6-20 \times 10^{-4} \mathrm{c} \mathrm{d}^{-1}$ on frequencies $F 1$ to $F 4$ respectively for NW Ser and $8-16 \times 10^{-4} \mathrm{c} \mathrm{d}^{-1}$ for V1446 Aql.

\subsection{NW Ser}

The rapid amplitude variation over the short timescale spanned by our observations strongly suggests the presence of a beating phenomenon. This would explain why after prewhitening for the first peak (seen at $\mathrm{F} 1=1.197 \mathrm{c} \mathrm{d}^{-1}$ in Fig. 2, together with its aliases at $\left.1,2, \ldots \mathrm{c} \mathrm{d}^{-1}\right)$ a second peak appears at a very close position corresponding to a frequency at $F 2=1.126 \mathrm{c} \mathrm{d}^{-1}$ (together with its daily aliases). Note that the width of the sidelobe is almost twice that calculated for the spectral window. This would mean that another frequency close to $1.197 \mathrm{c} \mathrm{d}^{-1}$ is also present. Following Loumos \& Deeming (1978), this occurs when the true frequencies differ by less than the half-width of the sidelobe and, in our case, a peak at the midpoint of the true frequencies would appear in the periodogram, as seen in Fig. 2. F1 and $F 2$ have comparable amplitudes, which would produce the rapid amplitude variability in terms of a beating phenomenon.

These two frequencies differ by less than the Rayleigh resolution, which is in our case $0.11 \mathrm{c} \mathrm{d}^{-1}$. This could indicate that the value we calculate for these frequencies might not be the real ones. However, the rapid amplitude variation present in the light curve, could hardly be explained by any other origin than the presence of two closely spaced frequencies, even if their real values are not exactly those given in Table 3 . Therefore we conclude that two close frequencies do actually exist, although their exact values might differ from those given here. In any case, Loumos $\&$ Deeming (1978) noted that the true frequencies always fall at the same midpoint as those found when the resolution is not high enough.

Residuals after prewhitening for these two frequencies have been analysed for additional periodic components. Frequencies $F 3=3.304 \mathrm{c} \mathrm{d}^{-1}$ and $F 4=1.412 \mathrm{c} \mathrm{d}^{-1}$ and their daily aliases have been detected in successive periodograms. These new frequencies have a much lower amplitude than $F 1$ and $F 2$, although they have a SNR greater than 4 , as shown in Fig. 2. Note that $F 3=3.304 \mathrm{c} \mathrm{d}^{-1}$ could be a combination of the two highamplitude frequencies $F 1$ and $F 2$, since $F 3 \sim F 1+F 2+1$. Finally, the four frequencies which minimise the residuals are those shown in Table 3 for the four Strömgren bands. No other significant peaks appear in the periodogram after the final prewhitening.

It is important to note that the four frequencies obtained independently for the different $v b y$ filters are the same within the error boxes. For the $u$ filter we found the $1 \mathrm{c} \mathrm{d}^{-1}$ alias of the lower amplitude frequencies $F 3$ and $F 4$. This is due to the low SNR of the data at that wavelength. As seen in Table 3 , the percentage of the total fraction of the variance that is removed ( $R$-values) for all filters is very high.

Note that the final frequencies can be contaminated by $1 \mathrm{c} \mathrm{d}^{-1}$ aliases, since the observations have been obtained at only one site. Keeping this in mind, if we compare with the frequencies found by other authors, the frequency $F 2$ could be a 1-day alias of the first frequency $\left(f 1=2.11 \mathrm{c} \mathrm{d}^{-1}\right)$ detected by Aerts (2000) using Hipparcos data and $F 4$ could be a 1-day alias of her second frequency $\left(f 2=2.46 \mathrm{c} \mathrm{d}^{-1}\right)$. In the same way, $F 1$ could be the 1-day alias of the frequency $2.17 \mathrm{c} \mathrm{d}^{-1}$ obtained by Percy et al. (1999). The complexity of the Hipparcos spectral window due to the few (76) datapoints spanning $\sim 1000$ days makes the detection of multiple short periods very difficult and uncertain. Nevertheless, to test our results we performed a reanalysis of the Hipparcos light curve with the non-linear leastsquare fitting code. We have detected the two frequencies found by Aerts (2000) as the best 2-frequency fitting. However, the best 3 -frequency model is given by the frequencies $2.09,1.43$ and $3.30 \mathrm{c} \mathrm{d}^{-1}$. The frequency $F 2$ could be the 1-day alias of $2.09 \mathrm{c} \mathrm{d}^{-1}$ and the two latter are similar to $F 4$ and $F 3$ respectively.

In Table 3 we list the frequencies resulting from the analysis of the OSN data. The above considerations indicate that these frequencies can be either real or in some cases their 1-day aliases. The analysis of the Hipparcos data shows the presence of the same frequencies or their aliases, confirming that NW Ser is 


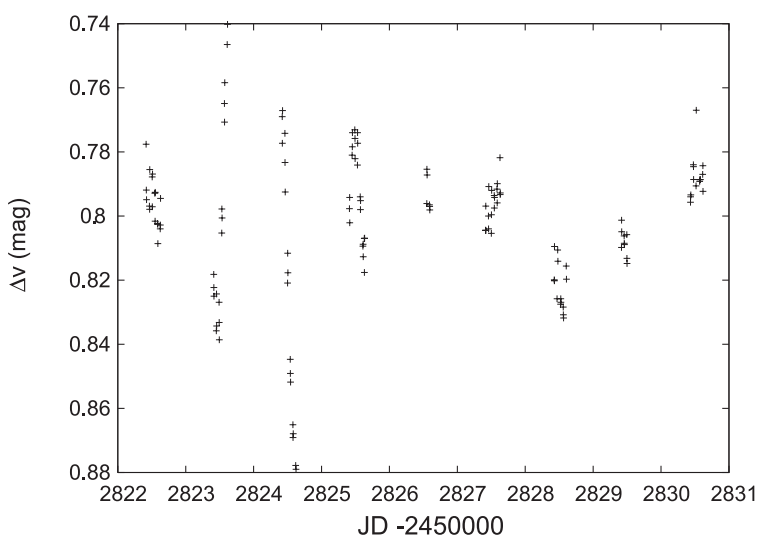

Fig. 3. Light curve of V1446 Aql in the $v$ filter.

a multiperiodic variable, as first suggested by Aerts (2000), and that the pulsation modes remain stable at least between the two epochs. To ascertain what frequencies are real or the aliases, a more extended photometric dataset with a better spectral window, either from a ground-based multisite campaign or from space, would be needed.

\subsection{V1446 AqI}

The light curve of V1446 Aql, presented in Fig. 3, also shows rapid variation which is probably caused by the presence of a beating phenomenon between two close frequencies. As the frequencies found for the four Strömgren filters are basically the same, we only discuss here the detailed analysis for the $v$ filter, since it shows the highest SNR. Frequencies at $1.617 \mathrm{c} \mathrm{d}^{-1}$ and at $2.783 \mathrm{c} \mathrm{d}^{-1}$ and their daily aliases appear clearly in the periodogram. Note that these two frequencies have similar amplitude. The amplitude of the first frequency $\left(1.617 \mathrm{c} \mathrm{d}^{-1}\right)$ fulfils the signal to noise criterion, as shown in Fig. 4. After prewhitening for this frequency, the second frequency at $2.783 \mathrm{c} \mathrm{d}^{-1}$, and its daily aliases, appear clearly. By prewhitening for these two frequencies, other components have been found. Either a frequency at $2.557 \mathrm{c} \mathrm{d}^{-1}$ or its 1-day alias at $1.557 \mathrm{c} \mathrm{d}^{-1}$ can be considered as the third frequency, although $1.557 \mathrm{c} \mathrm{d}^{-1}$ is so close to $F 1$ that the fitting algorithm does not converge and we obtain unrealistic values for this solution. Therefore we remove the frequency at $F 3=2.557$ and identify the fourth component $F 4=1.269 \mathrm{c} \mathrm{d}^{-1}$, or one of its aliases.

The final set of frequencies, displayed in Table 4, fulfils the signal to noise requirement in all the filters. We stopped the frequency search at this point because new peaks did not fulfil the SNR criterion mentioned above.

As in the previous subsection, we list the frequencies corresponding to the best fit to our OSN data. Due to the effects of data sampling discussed above, some of the listed frequencies might be 1-day aliases of the real ones.

\section{Theoretical modelling}

To determine whether the observed frequencies are predicted unstable or not by the current models of stellar pulsation we have made a preliminary theoretical study. The physical parameters of NW Ser and V1446 Aql have been obtained from the analysis of high resolution spectra (Frémat et al. 2006), taking into account the effects of gravitational darkening and assuming both stars are rotating at $88 \%$ of their critical break-up velocity (Frémat et al. 2005). The obtained values and associated errors are depicted in
Table 4. As for Table 3, but for V1446 Aql.

\begin{tabular}{|c|c|c|c|c|c|c|}
\hline No. & $\begin{array}{l}\begin{array}{l}\text { Freq. } \\
\mathrm{c} \mathrm{d}^{-1}\end{array} \\
\end{array}$ & $\begin{array}{l}\text { Amp. } \\
\text { mmag }\end{array}$ & $\begin{array}{l}\text { Phase } \\
2 \pi \mathrm{rad} \\
\end{array}$ & 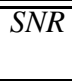 & $\begin{array}{c}\sigma_{\text {res }} \\
\text { mmag }\end{array}$ & $\begin{array}{l}R \\
\% \\
\end{array}$ \\
\hline & Filter $y$ & & $N=136$ & \multicolumn{3}{|c|}{$\sigma_{\text {init }}=23.89 \mathrm{mmag}$} \\
\hline$F 1$ & 1.631 & 21.1 & 0.76 & 17 & 19.6 & \\
\hline$F 2$ & 2.792 & 18.8 & 0.21 & 11 & 15.3 & \\
\hline$F 3$ & 2.559 & 18.7 & 0.66 & 12 & 12.3 & \\
\hline$F 4$ & 1.264 & 13.0 & 0.32 & 11 & 8.8 & 87 \\
\hline & Filter $b$ & & $N=136$ & \multicolumn{3}{|c|}{$\sigma_{\text {init }}=24.85 \mathrm{mmag}$} \\
\hline$F 1$ & 1.622 & 21.7 & 0.78 & 14 & 20.4 & \\
\hline$F 2$ & 2.783 & 20.9 & 0.22 & 11 & 15.1 & \\
\hline$F 3$ & 2.555 & 17.7 & 0.65 & 10 & 12.3 & \\
\hline$F 4$ & 1.266 & 12.9 & 0.33 & 8 & 8.9 & 87 \\
\hline & Filter $v$ & & $N=136$ & \multicolumn{3}{|c|}{$\sigma_{\text {init }}=24.84 \mathrm{mmag}$} \\
\hline$F 1$ & 1.617 & 22.1 & 0.79 & 15 & 19.9 & \\
\hline$F 2$ & 2.783 & 20.9 & 0.23 & 11 & 14.3 & \\
\hline$F 3$ & 2.557 & 17.5 & 0.64 & 10 & 11.7 & \\
\hline$F 4$ & 1.269 & 12.4 & 0.32 & 8 & 8.3 & 89 \\
\hline & Filter $u$ & & $N=136$ & \multicolumn{3}{|c|}{$\sigma_{\text {init }}=29.10 \mathrm{mmag}$} \\
\hline$F 1$ & 1.601 & 27.0 & 0.77 & 13 & 22.6 & \\
\hline$F 2$ & 2.759 & 25.5 & 0.32 & 11 & 17.4 & \\
\hline$F 3$ & 2.514 & 11.1 & 0.67 & 5 & 14.6 & \\
\hline$F 4$ & 1.187 & 15.5 & 0.62 & 8 & 11.1 & 85 \\
\hline
\end{tabular}

Fig. 5. Note that both error boxes are located in the overlapping region of the $\beta$ Cephei and SPB instability strips (Pamyatnykh 1999).

Evolutionary tracks computed with the numerical code CESAM (Morel 1997) are shown in Fig. 5. First-order effects of rotation are taken into account in the equilibrium models. To do so, the equilibrium equations are modified in the CESAM code in the manner described in Kippenhahn \& Weigert (1990). The so-called pseudo-rotating models include the spherically averaged contribution of the centrifugal acceleration by means of an effective gravity $g_{\text {eff }}=g-\mathcal{A}_{\mathrm{c}}(r)$, where $g$ is the local gravity, $r$ is the radius, and $\mathcal{A}_{\mathrm{c}}(r)=\frac{2}{3} r \Omega^{2}(r)$ is the centrifugal acceleration of matter elements. This spherically averaged component of the centrifugal acceleration does not change the order of the hydrostatic equilibrium equations. The models are assumed to rotate uniformly and to conserve their total angular momentum during evolution.

Considering the corresponding error boxes, masses in the range of 8.5-9.5 $M_{\odot}$ are found to be representative of NW Ser. Rotational velocities are in the range of 250 to $270 \mathrm{~km} \mathrm{~s}^{-1}$, radii from 5.8 to $7.9 R_{\odot}$ and ages around $20 \mathrm{Myr}$. In the same way, for V1446 Aql, a mass range of 7-7.5 $M_{\odot}$ is found, with rotational velocities ranging from 238 to $331 \mathrm{~km} \mathrm{~s}^{-1}$, radii from 4 to $4.5 R_{\odot}$ and an age that brings the star close to the ZAMS.

The instability analysis is then performed using the GraCo code (see Moya et al. 2004, for more details), which is based on the non-adiabatic equations derived by Unno et al. (1989). For both stars, the $\ell=2$ and $\ell=3$ modes are predicted to be unstable. In the case of NW Ser, the SPB and $\beta$ Cephei instability ranges are very close (Fig. 6), and this suggests that NW Ser is a good candidate to be a hybrid pulsator. This result is consistent with its location in the HR diagram region where the instability strips of SPB and $\beta$ Cephei overlap. In the case of V1446 Aql, only high-order g-modes with $\ell=2$ and 3 are predicted to be unstable close to the observed frequencies, as shown in Fig. 7.

In order to have an idea of what spherical degree $\ell$ is excited in these stars we have applied the photometric mode identification as described in Watson (1988) and used for $\delta$ Scuti stars in Garrido (2000). We have computed the amplitude ratios for each pulsational frequency and we have compared them with those 

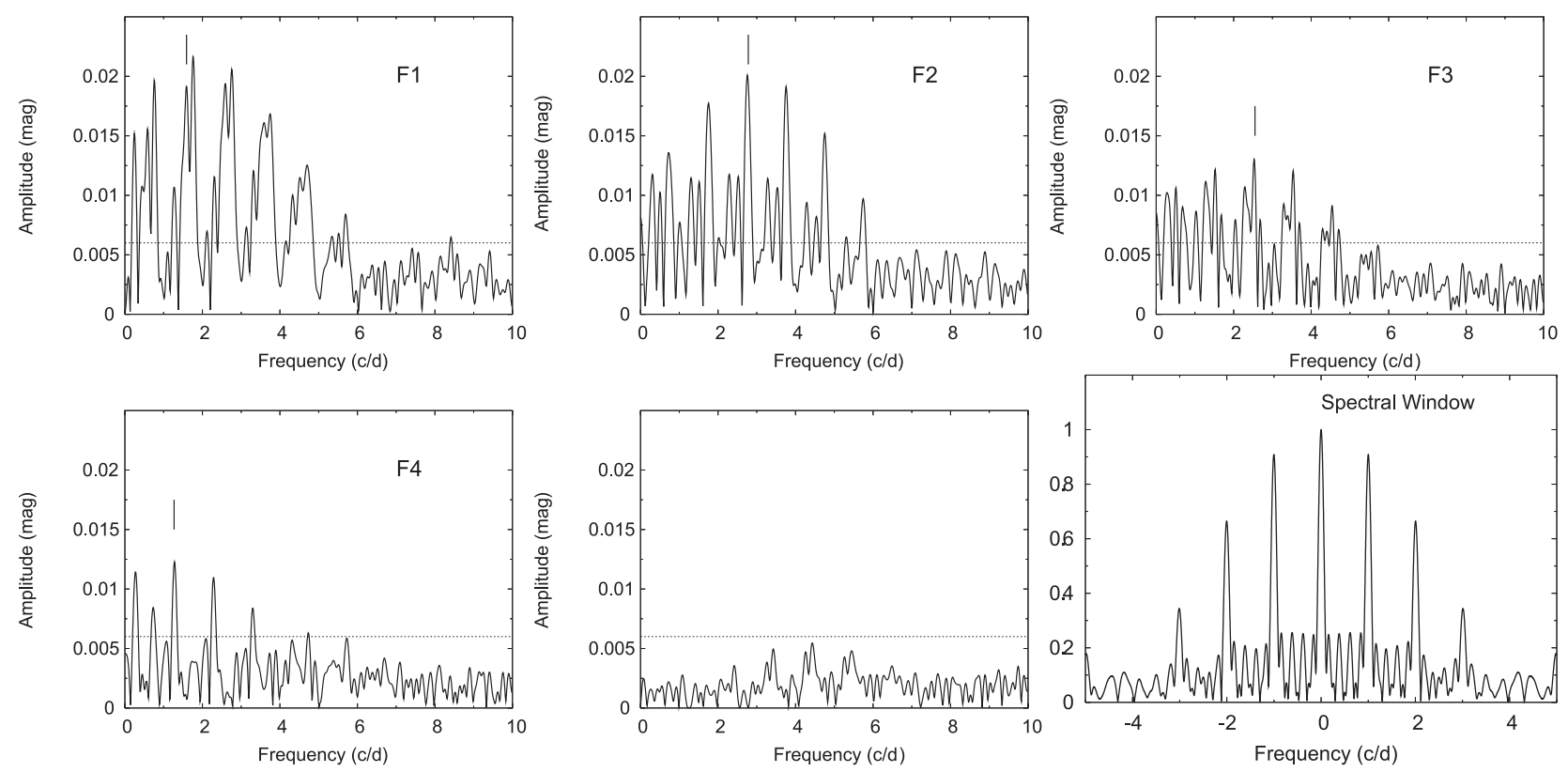

Fig. 4. Successive periodograms for V1446 Aql for the $v$ filter. Symbols are the same as for Fig. 2.

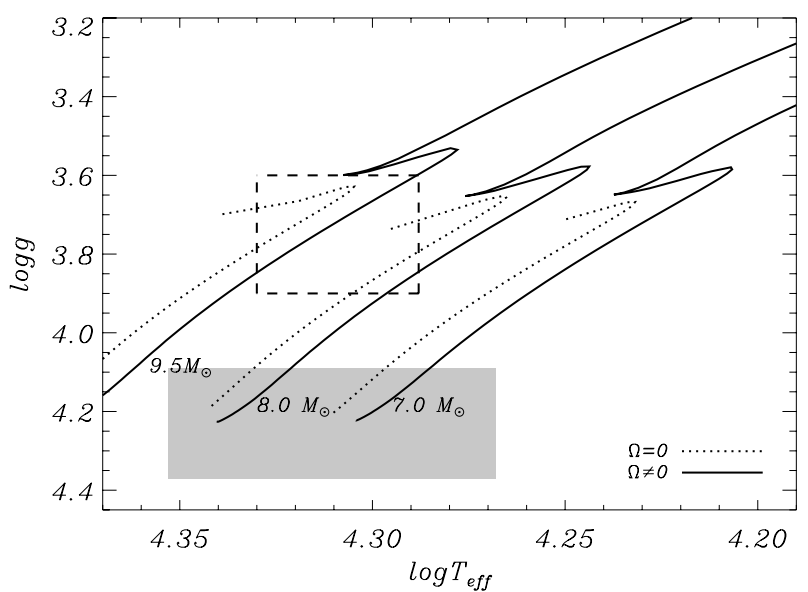

Fig. 5. Hertzsprung-Russel diagram showing the two Be stars considered in this study and some relevant evolutionary tracks selected for the modelling. The empty and shaded regions represent the corresponding error boxes for NW Ser and V1446 Aql respectively. Continuous and dotted lines represent evolutionary tracks of 7,8 and $9.5 M_{\odot}$ for rotating models and non-rotating models respectively.

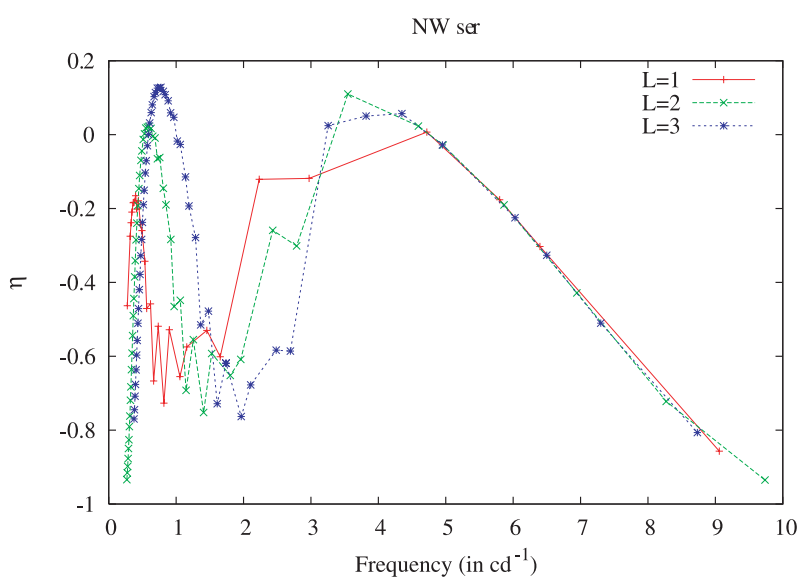

Fig. 6. Growth rates diagram for NW Ser: positive values indicate unstable modes and vice versa.

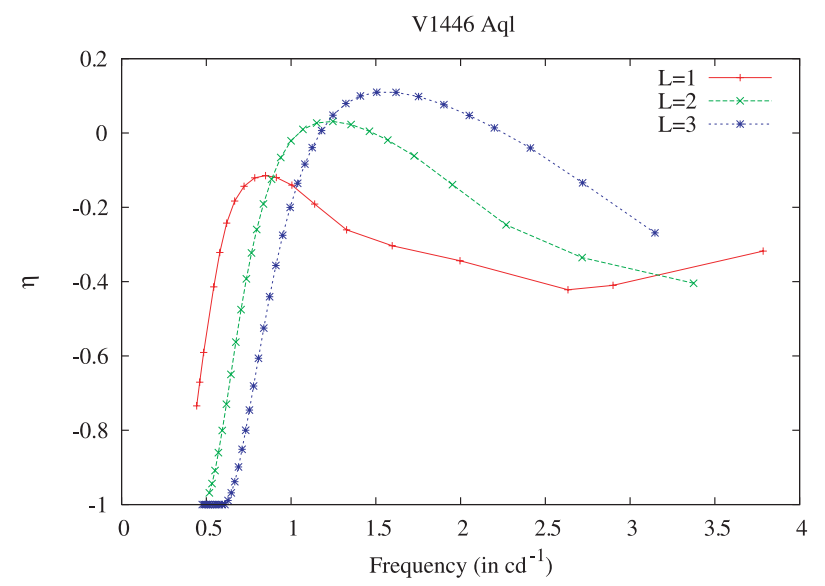

Fig. 7. Growth rates diagram for V1446 Aql.

predicted for stellar models using different $\ell$-degrees. In Figs. 8 and 9 we show the amplitude ratios for both stars. In the case of NW Ser, pulsation modes of degree $\ell=3$ appear to be more probable for frequencies $F 2$ and $F 4$, whereas for $F 1$ and $F 3$ the more probable degrees seem to be $\ell=1$ and 2. For V1446 Aql all detected frequencies seem to be associated with dipoles and quadrupoles. The $u$ amplitude for frequency $F 3$ is very low and probably due to the noise of the data at this wavelength.

At this point only spectroscopic observations can supply an unambiguous mode identification for these rapidly rotating objects.

\section{Conclusions}

Accurate photometric light curves of the Be stars NW Ser and V1446 Aql obtained during a preparatory program for the COROT space mission have been analysed. Our spectral analysis reveals the presence of four statistically significant frequencies in each star. Together with the multiperiodicity of HD 163868 and $\zeta$-Oph recently observed from space (Walker et al. 2005a,b), the present results point towards the 


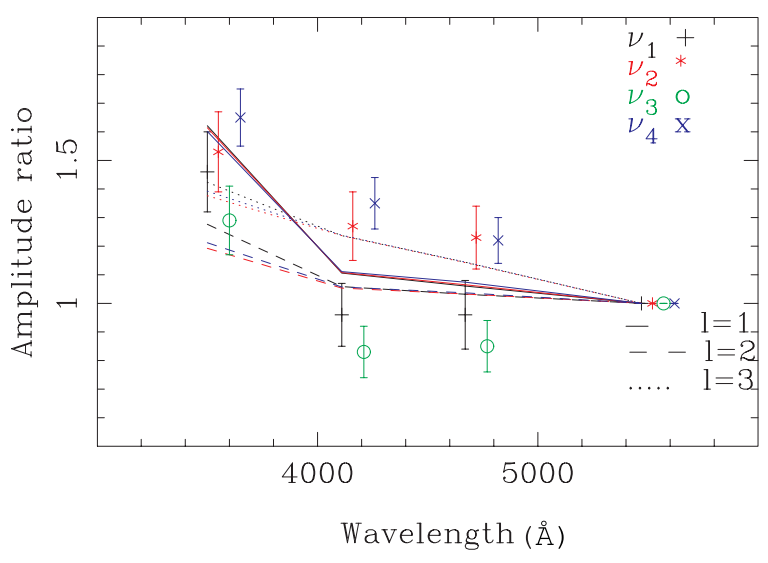

Fig. 8. Observed amplitude ratios $A_{X} / A_{y}$ and their error boxes for the detected frequencies of NW Ser. $A_{X}$ stands for any of the amplitudes in the $u v b y$ Strömgren filters. The lines represent the theoretical nonadiabatic predictions of the stellar models explained in the text.

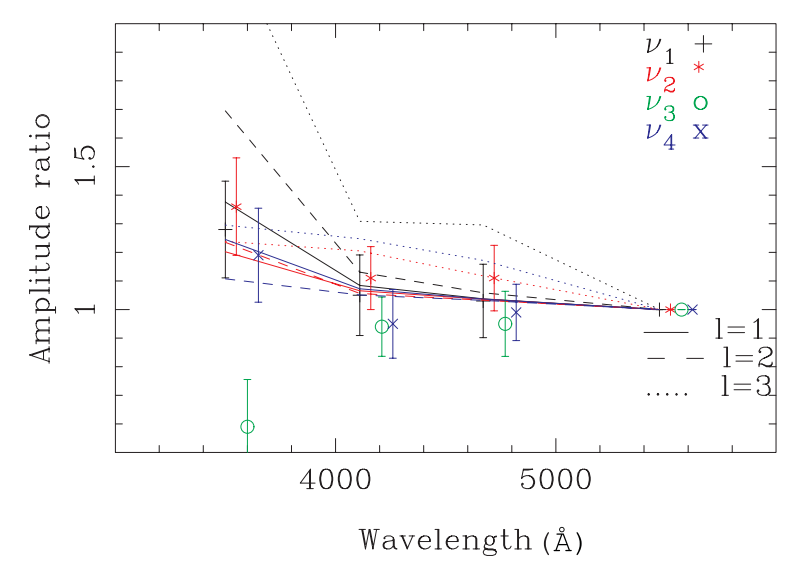

Fig. 9. Same as Fig. 8, but for V1446 Aql.

interpretation of short-period variability of Be stars in terms of $n r p$, as suggested by spectroscopic observations obtained in the last decade. In this framework, a theoretical instability analysis of the observed frequencies has been performed. Recent studies (Saio et al. 2007; Dziembowski et al. 2007) show that, for very rapid rotators, such as Be stars, the effect of rotation may also affect the instability of the modes. In future studies these effects should then be taken into account and compared with the present results. It is found that NW Ser could be an hybrid pulsator, since the ranges of unstable g-modes (SPB-like) and p-modes ( $\beta$ Cephei-like) are very close. This would thus imply that NW Ser is a good candidate of the very few stars detected so far showing such pulsational characteristics. In the case of V1446 Aql, only frequencies corresponding to predicted g-modes have been detected.

Longer timebase spectroscopic and photometric observations are thus required in order to confirm these results.

Acknowledgements. This research is based on data obtained at the Observatorio de Sierra Nevada, which is operated by the CSIC through the Instituto de Astrofísica de Andalucía. The work of J.G-S. is supported by a FPU grant from the Spanish "Ministerio de Educacion y Ciencia". J.F. and J.S. acknowledge financial support from the program ESP 2004-03855-C03. J.C.S acknowledges support by the Instituto de Astrofísica de Andalucía by and I3P contract financed by the European Social Fund and from the Spanish Plan Nacional del Espacio under project ESP2004-03855-C03-C01.

\section{References}

Aerts, C. 2000, in IAU Coll. 175: The Be Phenomenon in Early-Type Stars, ASP Conf. Ser., 214, 192

Baade, D. 1982, A\&A, 105, 65

Baglin, A., Auvergne, M., Catala, C., et al. 2002, in IAU Coll. 185, Radial and Nonradial Pulsationsn as Probes of Stellar Physics, ed. C. Aerts, T. R. Bedding, \& J. Christensen-Dalsgaard, ASP Conf. Ser. 259, 626

Breger, M., Stich, J., Garrido, R., et al. 1993, A\&A, 271, 482

Dziembowski, W. A., Daszyńska-Daszkiewicz, J., \& Pamyatnykh, A. A. 2007, MNRAS, 374, 248

Frémat, Y., Neiner, C., Hubert, A.-M., et al. 2006, A\&A, 451, 1053

Frémat, Y., Zorec, J., Hubert, A.-M., \& Floquet, M. 2005, A\&A, 440, 305

Garrido, R. 2000, in Delta Scuti and Related Stars, ASP Conf. Ser., 210, 67

Hubert, A. M., \& Floquet, M. 1998, A\&A, 335, 565

Kazarovets, A. V., Samus, N. N., Durlevich, O. V., et al. 1999, Inform. Bull. Variable Stars, 4659, 1

Kippenhahn, R., \& Weigert, A. 1990, Stellar structure and evolution, A\&A library (Springer-Verlag)

Lenz, P., \& Breger, M. 2005, Commun. Asteroseismol., 146, 53

Loumos, G. L., \& Deeming, T. J. 1978, Ap\&SS, 56, 285

Maintz, M., Rivinius, T., Štefl, S., et al. 2003, A\&A, 411, 181

Merrill, P. W., \& Burwell, C. G. 1943, ApJ, 98, 153

Montgomery, M., \& O’Donoghue, D. 1999, Delta Scuti Newsletter, 13, 28

Morel, P. 1997, A\&AS, 124, 597

Moya, A., Garrido, R., \& Dupret, M. A. 2004, A\&A, 414, 1081

Neiner, C., Floquet, M., Hubert, A. M., et al. 2005, A\&A, 437, 257

Pamyatnykh, A. A. 1999, Acta Astron., 49, 119

Percy, J. R., Marinova, M. M., Božić , H., \& Harmanec, P. 1999, A\&A, 348, 553

Rivinius, T., Baade, D., Štefl, S., et al. 2001, A\&A, 369, 1058

Saio, H., Cameron, C., Kuschnig, R., et al. 2007, ApJ, 654, 544

Schwarzenberg-Czerny, A. 1991, MNRAS, 253, 198

Unno, W., Osaki, Y., Ando, H., Saio, H., \& Shibahashi, H. 1989,

(Tokyo: University of Tokyo Press), 2nd Ed.

Vaníček, P. 1971, Ap\&SS, 12, 10

Walker, G. A. H., Kuschnig, R., Matthews, J. M., et al. 2005a, ApJ, 635, L77

Walker, G. A. H., Kuschnig, R., Matthews, J. M., et al. 2005b, ApJ, 623, L145

Watson, R. D. 1988, Ap\&SS, 140, 255

Zerbi, F. M., Garrido, R., Rodriguez, E., et al. 1997, MNRAS, 290, 401 